\title{
IMPACTO FAMILIAR DE LA SEDACIÓN PALIATIVA EN PACIENTES TERMINALES DESDE LA PERSPECTIVA DEL CUIDADOR PRINCIPAL
}

\author{
FAMILY IMPACT OF PALLIATIVE SEDATION IN TERMINALY ILL FROM THE \\ PERSPECTIVE OF THE MAIN CAREGIVER
}

\begin{abstract}
Ana María Carmona Jiménez , Melina González², María Clara Vélez Ángel y Alicia Krikorian $^{3}$

1 Hospital Universitario San Vicente de Paul, Medellín, Colombia.

2 Facultad de Medicina, Escuela de Ciencias de la Salud, Universidad Pontificia Bolivariana, Medellín, Colombia.

${ }_{3}^{3}$ Grupo de Dolor y Cuidado Paliativo, Escuela de Ciencias de la Salud, Universidad Pontificia Bolivariana, Medellín, Colombia.
\end{abstract}

Resumen

Objetivo: Describir el impacto familiar de la sedación paliativa en pacientes con enfermedad avanzada terminal ya fallecidos, desde la percepción del cuidador principal.

Método: Estudio cualitativo de enfoque fenomenológico. Se realizaron entrevistas semiestructuradas a diez cuidadores principales de pacientes con enfermedad terminal fallecidos bajo sedación paliativa, que fueron atendidos por cuidados paliativos en una institución oncológica de Medellín, Colombia entre enero y marzo de 2015. Se analizó la información a partir de categorías preestablecidas: significado, cambios emocionales, cambios estructurales y duelo.

Resultado: Los cuidadores no tenían ningún conocimiento previo sobre la sedación. Todos reportan que fueron informados sobre el objetivo y los cambios físicos que podía presentar el paciente; sin embargo, la mitad de ellos refirieron que esta información no fue suficiente para comprender el proceso que enfrentaban. Manifestaron diversos sentimientos (alivio, duda, impotencia, tristeza) antes, durante y después de la sedación. Respecto a los cambios estructurales, la mayoría de las familias se reorganizaron y facilitaron el acompañamiento y cuidado al paciente refiriendo fortalecimiento de las rela-
Abstract

Objective: The present study aimed at describing the impact that palliative sedation had on the family and their subsequent bereavement process, from the primary caregiver's point of view.

Methodology: Qualitative study with a phenomenological approach; ten primary caregivers of patients with terminal illness who were treated by the palliative care group of an oncological institution in Medellin, Colombia and died under palliative sedation were interviewed. The information was analyzed using predetermined categories: meaning, emotional changes, structural changes and bereavement.

Results: Caregivers had no prior knowledge about palliative sedation. All of them reported to be informed about the possible changes that patients could go through. However, half of the caregivers reported that the information was insufficient to understand the process they were facing. They expressed multiple feelings (relief, doubt, helplessness and sadness) before, during and after palliative sedation. Regarding structural changes, all families underwent reorganization, and family relationships were strengthened. On the whole, caregivers perceived that palliative sedation facilitated

Correspondencia:

Alicia Krikorian. Universidad Pontificia Bolivariana, Campus de Robledo. Calle $78 b$ No. 72a-109, Medellín, Colombia.

E-mail: aliciakriko@gmail.com 
ciones familiares. Finalmente, se encontró que los cuidadores percibieron que la sedación paliativa facilita el duelo, al aliviar el sufrimiento y permitiendo una buena muerte.

Conclusiones: Los cuidadores evalúan la experiencia de sedación como positiva y satisfactoria para aliviar el sufrimiento de su ser querido y el de su familia. Igualmente, facilita el proceso de duelo por la construcción positiva de alivio en torno a la experiencia vivida como familia, fortaleciendo sus relaciones y recordando una buena muerte para su paciente.

Palabras clave: Cuidados paliativos, cuidado terminal, sedación, cuidador, familia, duelo. bereavement while relieving suffering and allowing a good death.

Conclusions: Caregivers considered that the experience of palliative sedation was positive and satisfactory, allowing to relief suffering of their loved one and the family. It also eased the bereavement process as families made a positive reconstruction of the experience, strengthening their relationships and leaving a good memory of the patient's death.

Keywords: Palliative care, terminal care, deep sedation, caregiver, family, bereavement.

\section{INTRODUCCIÓN}

El cuidado paliativo busca el cuidado integral de los pacientes que enfrentan enfermedades amenazantes para la vida ${ }^{(1-4)}$. Este implica paliar el sufrimiento al final de la vida. Para ello se cuenta con la Sedación Paliativa (SP) como opción terapéutica, la cual implica la administración de fármacos para lograr el alivio, que por otras medidas no ha sido posible, del sufrimiento físico $\mathrm{y} / \mathrm{o}$ psicológico, mediante la disminución de la consciencia lo suficientemente profunda y previsiblemente irreversible en un paciente cuya muerte está muy próxima ${ }^{(5)}$. La SP está indicada para el manejo de síntomas refractarios ${ }^{(6,7)}$, cuando no ha habido una respuesta adecuada al tratamiento paliativo correcto e intenso sin comprometer el estado de conciencia del paciente ${ }^{(5)}$. Entre los síntomas refractarios más frecuentes se encuentran: delirium, dolor, disnea y distrés psicológico ${ }^{(8)}$. A pesar de ello y de su clara indicación actual, la SP continúa generando debates por las posturas a favor y en contra, así como cuestionamientos éticos al comparla con la eutanasia ${ }^{(7,9)}$.

La toma de decisiones respecto a la SP debe realizarse contando con el consentimiento implícito o explícito del paciente, quien puede solicitarla y analizarla en conjunto con su médico tratante. También se puede usar el consentimiento delegado, donde la familia asume la toma de decisión con el equipo sanitario cuando el paciente no está en condiciones para decidir de forma autónoma. No obstante, la decisión final de sedar a un paciente en fase terminal es un acto médico que incluye una evaluación cuidadosa de los síntomas, un establecimiento de objetivos y fármacos a utilizar y un énfasis en el alivio del sufrimiento como $\operatorname{meta}^{(10,11)}$.

Para la realización de la SP se requiere el papel activo de la familia para la toma de decisión cuando el paciente no puede hacerlo. Una de las mayores necesidades de los familiares es la constante comunicación con el personal médico y la disposición del soporte psicosocial pertinente ${ }^{(12)}$. La SP puede ser fuente de preocupaciones para los pacientes, familiares y cuidadores, recayendo en ellos sus consecuencias tanto positivas como negativas; por tanto, se requiere mayor atención e intervención de los profesionales de cuidados paliativos ${ }^{(13)}$.

En un estudio realizado por Van Dooren et al. ${ }^{(14)}$, el $51 \%$ de los familiares expresó preocupación frente al objetivo de la sedación luego de haber sido iniciada, así como 
sobre el bienestar del paciente y de ellos mismos como cuidadores. Se concluyó que los familiares están en necesidad de información continua y acompañamiento profesional durante la SP, pues este proceso los acerca a la muerte del paciente. Por su parte, Morita et al. ${ }^{(10)}$ encontraron que el $50 \%$ de las preocupaciones de las familias con pacientes oncológicos en SP estaban relacionadas con no poder comunicarse con el paciente, $34 \%$ no estaban preparados para los cambios en la condición del paciente y $28 \%$ manifestaba carga con la responsabilidad de la decisión. Particularmente, algunas familias manifestaron no encontrar sentido en acompañar al paciente en este estado. Sin embargo, disminuyó el malestar emocional general respecto a la situación de terminalidad luego de comenzar la SP. Este estudio sugirió que la atención a las necesidades familiares se debía centrar en el manejo y acompañamiento de síntomas emocionales, en la constante comunicación entre el personal sanitario y la familia y en el compartir y acompañar la toma de decisión.

En una revisión sistemática que incluyó 36 estudios sobre la experiencia de los familiares con la $\mathrm{SP}^{(15)}$, se encontró que la mayoría de ellos expresaban satisfacción con la terapia. Sin embargo, diez investigaciones encontraron un alto grado de malestar emocional en los familiares frente a esta práctica, relacionado con sentir carga frente a la decisión tomada, percibir poca compasión por parte del personal médico, contar con poca preparación para los cambios que iban a presenciar en el paciente y el corto período entre la sedación y la muerte. Entre las reacciones emocionales y cognitivas que mediaban el malestar emocional estaban la ira, la frustración, el desacuerdo, la culpa, la impotencia, las preocupaciones, los conflictos y el cansancio, tanto emocional como físico. Otros resultados importantes indican que entre el $78 \%$ y el $93 \%$ de los familiares reportan satisfacción con la SP puesto que sienten que el sufrimiento en sus seres que- ridos ha acabado y que se les esta dando el mayor confort posible para una muerte tranquila. Ninguna investigación reportó explícitamente haber realizado un soporte o apoyo a los cuidadores durante o después de la SP, situación que implica un reto para los profesionales de los cuidados paliativos.

Generalmente, las familias están presentes antes de la muerte del paciente y están directamente implicadas en su cuidado; por tanto, se pueden ver afectadas por las decisiones del tratamiento y sus efectos $^{(13,16-18)}$. El cuidado del enfermo se hace más demandante y extenuante para el cuidador principal; a esto se suma el malestar emocional, físico, social y espiritual que el proceso de morir y la toma de conciencia sobre la muerte traen consigo ${ }^{(19,20)}$. Dicha experiencia implica para los cuidadores, pensar y asumir la continuidad de la vida, así como reorganizar y restablecer roles.

En el contexto Latinoamericano no se han realizado, hasta el momento, estudios sobre el impacto familiar de la SP, por lo que se desconocen sus reacciones emocionales ante ésta decisión, la influencia de sus creencias, los cambios familiares a nivel estructural y de qué manera favorece o dificulta su proceso de duelo ${ }^{(21-24)}$. En consecuencia, el objetivo del presente estudio es describir el impacto familiar desde la percepción que tiene el cuidador principal sobre la experiencia de la SP en pacientes ya fallecidos y que fueron atendidos en la unidad de cuidados paliativos de una institución oncológica. Previamente se realizó una revisión teórica sobre el impacto de la enfermedad avanzada y su significado en la familia ${ }^{(27)}$, los cambios estructurales y emocionales familiares ${ }^{(21)}$ y el proceso de duelo ${ }^{(24)}$.

\section{MÉTODO}

\section{Tipo de estudio y participantes}

Estudio cualitativo de enfoque fenomenológico. En el estudio participaron 10 cui- 
dadores principales familiares de pacientes con enfermedad terminal que fallecieron bajo SP, atendidos por el equipo de cuidados paliativos de una institución oncológica de la ciudad de Medellín, Colombia entre enero y marzo de 2015, y quienes fueron seleccionados a través de un muestreo intencional hasta lograr saturación de la información. Los cuidadores fueron identificados personalmente por el equipo tratante o telefónicamente al indagar quién ejercía dicho rol. Criterios de inclusión: ser cuidador familiar, mayor de 18 años, residente en Medellín o el área metropolitana y aceptar participar voluntariamente mediante consentimiento informado firmado. Se excluyeron aquellos cuidadores familiares con significativo malestar emocional al momento del contacto inicial, con el fin de minimizar el riesgo psicológico o la generación de mayor sufrimiento durante la entrevista del ya asumido como parte del proceso normal de duelo.

\section{Instrumento}

Se diseñó una guía de preguntas para la realización de la entrevista semi-estructurada (Anexo 1) que exploraba las opiniones, percepciones $y / 0$ experiencias y permitió obtener información a profundidad sobre el tema. La primera parte incluía la recolección de datos sociodemográficos del cuidador principal, el paciente fallecido y la conformación familiar. La segunda parte correspondió a preguntas abiertas realizadas exclusivamente al cuidador frente a su percepción del impacto familiar producido por la SP. Se preestablecieron las siguientes categorías de análisis: (1) comprensión familiar de la SP, (2) cambios emocionales en la familia, (3) los cambios estructurales y (4) proceso de duelo. En el análisis se tuvo en cuenta la aparición de categorías emergentes, sin embargo no se identificó ninguna durante las entrevistas.

\section{Procedimiento}

El proyecto fue aprobado por el comité de ética de la Universidad Pontificia Bolivariana y de la institución oncológica participante. Los médicos del equipo de cuidados paliativos, encargados de realizar la SP, comentaron la finalidad del estudio a los cuidadores principales de los pacientes que se encontraban bajo sedación y los invitaron a participar. Una vez los participantes dieron su consentimiento verbal de participación se les informó que serían contactados telefónicamente por una investigadora (AMC) para ampliar información del estudio.

Se les contactó entre cuatro a seis semanas después del fallecimiento del paciente, para dar tiempo a la elaboración inicial del duelo y a la disminución del impacto emocional asociado al fallecimiento. La investigadora les invitó de nuevo a participar del estudio y se indagó por su estado emocional del momento para detectar factores de riesgo psicológico, empleando preguntas como: ¿Cómo está sintiendo con la muerte de su ser querido?, ¿ $\mathrm{Ha}$ percibido cambios emocionales importantes en las últimas semanas que le estén imposibilitando hacer sus actividades diarias?, ¿Le genera malestar emocional hablar de la experiencia como cuidador? Con aqueIlos cuidadores que reiteraron su deseo de participar y en los que no se detectaron factores de riesgo psicológico, se concertó un lugar y fecha de entrevista convenientes. El día de la entrevista se procedió a la firma del consentimiento informado, y se pidió autorización para grabarla en audio, antes de su inicio.

\section{Análisis de la información}

Se hizo una transcripción textual de las entrevistas y se elaboró una matriz de análisis según las categorías preestablecidas. El análisis se realizó teniendo en cuenta 
los objetivos del estudio, estableciendo semejanzas y diferencias en las respuestas dadas por los participantes.

\section{RESULTADOS}

\section{Caracterización sociodemográfica, clínica y familiar}

La mayoría de los cuidadores entrevistados eran mujeres, de religión católica y con una edad promedio de 55 años (rango
33-73), la mitad tenían estudios superiores y eran cónyuges del paciente; en más de la mitad de los casos eran viudas dedicadas a las labores del hogar. La composición familiar permitió detectar el tipo de familia y la etapa del ciclo vital. En la tipología familiar se encontró diversidad de las mismas. Las etapas del ciclo vital por las cuales atravezaban las familias de los cuidadores fueron, en mayor proporción, salida de los hijos/ nido acogedor (ver la tabla 1 para detalles).

\section{Tabla 1. Datos sociodemográficos}

\begin{tabular}{|c|c|}
\hline Variable / Categoría & $N(\%)$ \\
\hline $\begin{array}{l}\text { Escolaridad } \\
\qquad \begin{array}{l}\text { Estudios primarios } \\
\text { Estudios secundarios } \\
\text { Estudios superiores }\end{array}\end{array}$ & $\begin{array}{l}4(40) \\
1(10) \\
5(50)\end{array}$ \\
\hline $\begin{array}{l}\text { Relación cuidador - paciente } \\
\text { Conyuge } \\
\text { Hermana } \\
\text { Madre } \\
\text { Hija }\end{array}$ & $\begin{array}{l}5(50) \\
2(20) \\
2(20) \\
1(10)\end{array}$ \\
\hline $\begin{array}{l}\text { Estado civil } \\
\text { Viudo } \\
\text { Casado } \\
\text { Soltero }\end{array}$ & $\begin{array}{l}6(60) \\
3(30) \\
1(10)\end{array}$ \\
\hline $\begin{array}{l}\text { Ocupación } \\
\qquad \begin{array}{l}\text { Labores del hogar } \\
\text { Empleado }\end{array}\end{array}$ & $\begin{array}{l}6(60) \\
4(40)\end{array}$ \\
\hline $\begin{array}{l}\text { Religión } \\
\qquad \begin{array}{l}\text { Católica } \\
\text { Cristiano adventista }\end{array}\end{array}$ & $\begin{array}{l}9(90) \\
1(10)\end{array}$ \\
\hline $\begin{array}{l}\text { Tipología familiar } \\
\text { Monoparental } \\
\text { Nuclear } \\
\text { Extensa } \\
\text { Ampliada } \\
\text { Simultánea }\end{array}$ & $\begin{array}{l}3(30) \\
3(30) \\
2(20) \\
1(10) \\
1(10)\end{array}$ \\
\hline $\begin{array}{l}\text { Etapa del ciclio vital } \\
\text { Salida de los hijos/nido acogedor } \\
\text { Edad escolar } \\
\text { Adolescencia } \\
\text { Infancia } \\
\text { Plataforma/salida de los hijos }\end{array}$ & $\begin{array}{l}4(40) \\
2(20) \\
2(20) \\
1(10) \\
1(10)\end{array}$ \\
\hline
\end{tabular}


Los pacientes cuidados tuvieron los siguientes diagnósticos, según el reporte de los cuidadores: cáncer de mama (30\%), cáncer de pulmón (30\%), y otros tipos de cáncer (leucemia, glioblastoma, cérvix, gástrico, 40\%). Todos los pacientes recibieron al menos dos tratamientos oncoespecificos como cirugía, quimioterapia y radioterapia.

\section{Categorías de análisis}

\section{Comprensión familiar de la SP}

Esta categoría incluye aspectos relacionados con la comprensión de la SP: su definición, explicaciones recibidas por parte del equipo asistencial, satisfacción con su aplicación y expectativas frente al objetivo de la sedación (ver las respuestas de los cuidadores en la tabla 2).

En cuanto al concepto y conocimiento previo sobre la SP, la mayoría (9 de 10 cuidadores), no lo tenían. Con respecto a la comunicación_del personal de salud hacia los cuidadores, los 10 cuidadores reportaron haber recibido información sobre la opción de la sedación, no al comien- zo del diagnóstico, ni de su tratamiento, sino al final del proceso de enfermedad, cuando la expectativa de vida era de días. Un aspecto relevante en el significado de la sedación para los cuidadores fue comprender que se utilizaría un medicamento que disminuía el nivel de conciencia del paciente y cuyo objetivo era aliviar y controlar síntomas refractarios que generaban sufrimiento. Sin embargo, un cuidador refirió no haber recibido información clara sobre los efectos permanentes de la sedación y de la imposibilidad de comunicarse con el paciente, lo cual causo gran impacto emocional.

Todos los cuidadores refirieron haber recibido información sobre los cambios físicos que el paciente podría presentar posterior a la sedación, tales como: estado de sueño profundo, no poder comunicarse, no sentir hambre, ni sed y no controlar esfínteres. Aún así, cinco cuidadores manifestaron que la información brindada antes de la sedación no fue suficiente, dado que se presentaron otras situaciones inesperadas (p.ej., despertarse en medio de la sedación), lo que favoreció en ellos preocu-

\section{Tabla 2. Comprensión familiar de la SP}

\begin{tabular}{|c|c|}
\hline $\begin{array}{l}\text { Concepto y } \\
\text { conocimiento } \\
\text { previo sobre } \\
\text { la SP }\end{array}$ & $\begin{array}{l}\text { "No, fue algo totalmente nuevo, y ahí agradecí enormemente eso, porque } \\
\text { sabíamos que se iban a poder evitar muchos dolores de ella y para muchas } \\
\text { otras personas también. No es una eutanasia, es un buen morir, porque se evitan } \\
\text { muchos dolores tanto para el paciente como para uno." (CF6) }\end{array}$ \\
\hline $\begin{array}{l}\text { Comunicación } \\
\text { del personal } \\
\text { de salud }\end{array}$ & $\begin{array}{l}\text { "En las últimas semanas le aplicaron morfina y hubo poca tolerancia y el dolor } \\
\text { persistía, a partir de ahí viendo que ya no habían esperanzas de vida, entonces } \\
\text { hubo la propuesta de tomar la opción de la sedación." (CF4) }\end{array}$ \\
\hline $\begin{array}{l}\text { Comprensión } \\
\text { de la sedación }\end{array}$ & $\begin{array}{l}\text { "Nos explicaron a los dos, que no era para todos los pacientes, que era para } \\
\text { pacientes con enfermedad avanzada, progresiva y sin solución y segundo con } \\
\text { un síntoma que ya nada lo controlaba y que le estaba deteriorando la calidad } \\
\text { de vida a la persona, nos explicó que era una decisión propia que ponen el } \\
\text { medicamento, te duermen y tu antes de eso te despides de tu familia, porque no } \\
\text { vuelves posiblemente a despertar." (CF8) } \\
\text { "Pues para que ella no sintiera dolor, pero no me explicaron que ella ya no iba } \\
\text { a despertar más para haberme despedido de ella y que ella también lo hiciera." } \\
\text { (CF9) }\end{array}$ \\
\hline
\end{tabular}




\begin{tabular}{ll}
\hline Información & "El cambio fue que estaba más tranquila, no se alimentaba, se le quitaron los \\
& ronquidos al respirar, los labios muy secos, pero no se le podía dar agua porque \\
& eso la podía empeorar. Solo hubo un día que le pasó el efecto de la sedación \\
& y se despertó muy agitada, pero le dieron otra vez la droga y ya no se volvió a \\
& despertar asín. (CF9) \\
& "Pensé que él se iba a quedar dormido y ya, pero el intentó como despertar, \\
& muy angustiado, no se le entendía nada, quería tomar agua, yo no sabía qué \\
& hacer en ese momento, no pensé que eso podía pasar, en ese momento perdí la \\
& credibilidad en el proceso, porque en ningún momento me hablaron de eso y yo \\
& me asusté mucho. Entonces ahí la enfermera fue a explicarnos que eran varias \\
& dosis y que eso podía ir pasando y que se iba mirando cuanto se iba poniendo \\
& del medicamento." (CF8) \\
Expectativas de & "Las expectativas de nosotros eran que mi mamá no sufriera y que tuviera \\
los familiares & una muerte tranquila, que no la viéramos sufriendo hasta el último segundo \\
de su vida, que se fuera muy lentamente pero sin sufrimiento porque sabemos & que estas enfermedades son supremamente dolorosas. Que pudiéramos estar \\
tranquilos con ella, hablándole y acompañándola hasta el final". (CF7) \\
"No, no dio tiempo. A ella le empezaron la sedación como a la una y media o \\
dos de la tarde, y ella murió a las siete de la noche. Esas horas fueron terribles, \\
muy duras." (CF1)
\end{tabular}

paciones por el bienestar del paciente $y$ dudas relacionadas con el objetivo de la sedación.

Respecto a las expectativas de los familiares, se encontró que nueve cuidadores percibieron cumplimiento de la expectativa de aliviar el sufrimiento de su familiar. Un cuidador percibió que el alivio del sufrimiento no se cumplió, considerando que la aplicación de la SP fue tardía.

\section{Cambios emocionales en la familia}

Se exploraron los cambios emocionales en los cuidadores y sus familiares haciendo énfasis en tres momentos: antes, durante y después de la SP (ver la tabla 3).

Al momento de recibir la propuesta por el médico especialista, cinco cuidadores y sus familias sintieron alivio al percibirla como una alternativa terapéutica; dos cuidadores y sus familias tuvieron respuestas ambivalentes de alivio y tristeza: la primera por lograr paliar el sufrimiento de su fami- liar y la segunda frente a la muerte cercana que ello implicaba; y tres cuidadores y sus familiares sintieron duda e impotencia por la muerte cercana y se preguntaron si era la mejor decisión para su familiar.

Durante el proceso de sedación cinco cuidadores refirieron seguir sintiéndose aliviados y tranquilos; tres manifestaron impotencia y preocupación ante el estado de inconsciencia y la dificultad en la comunicación con el paciente; y dos manifestaron duda y temor relacionado con la decisión.

Con respecto a los sentimientos posteriores a la muerte del paciente, nueve cuidadores refirieron sentirse tranquilos en el momento de la entrevista con la decisión de la sedación y la evaluaron como una experiencia satisfactoria. Sin embargo, un cuidador manifestó que fue una alternativa terapéutica que logró aliviar el sufrimiento de su ser amado, aunque aún al momento de la entrevista experimentaba dudas sobre la decisión y temor de que hubiera sido una eutanasia. 


\title{
Tabla 3. Cambios emocionales en la familia ante la sedación paliativa
}

\begin{abstract}
Al inicio
"Alivio, sentí mucho alivio. Era un proceso muy duro para ella y para uno y entendimos que ya no había más opción." (CF6)

"Uno no sabe si es algo bueno o malo, a mí me parece terrible la idea de que uno tenga que decidir casi que cuando morirse, pero también estábamos en un momento en que mi esposo tenía un síntoma sin controlar (...) comenzaron a sedarlo parcialmente para que pudiera descansar. Son sentimientos encontrados, uno que bueno que bueno que te digan que hay una opción para dormir, para eliminarte esa carga para él y para mí, porque era horrible; pero cuando te dicen tú decides cuando no te despiertes más, escuchar eso es horrible, porque es tomar la decisión de cuando uno se va." (CF8)

"Pues yo si me sentí muy mal porque yo hubiera querido que ella me siguiera hablando, seguir conversando, porque ya era como con un muerto ahí, era como estarla velándola." (CF1)
\end{abstract}

Durante el "Muy bien, tranquilo. Fue lo mejor que se pudo hacer por ella. Si no se hubiera proceso sedado, no la hubiera querido verla mal, porque a ella el oxígeno ya no le valía, hubiera sido una muerte más dolorosa y de más sufrimiento para mí." (CF6)

"Preocupados porque ella estuvo muchos días así, parecía una muerta así, ella duro mucho sedada, nosotros pensamos que iba a ser menos tiempo." (CF9)

"Con dudas, yo no sabía si estaba obrando bien y era lo mejor para él, pero solo Dios sabe que yo lo acepte con todo el amor del mundo para que él estuviera bien y tuviera una muerte más tranquila, sin tanto dolor." (CF3)

Posterior a

"Yo me siento bien y tranquila, y yo sé que mi hermano también, yo no aprobé la muerte que le hicieran nada malo a mi mamá por el contrario que pudiera estar mejor y más tranquila, a veces lo hablamos mucho y pensamos que fue lo mejor que ella no tuviera más sufrimiento, porque ella tuvo una época con mucho dolor y sufrimiento." (CF6)

"Yo en un principio me sentí tranquila. Pero después (...) comentábamos sobre un miedo en relación con una mala decisión; pensando en lo de la eutanasia, que de pronto le aligeramos. Se pensó en eso, pero en la situación que estaba ella, que no había esperanza de vida en absoluto, entonces no, tranquila." (CF1)

\section{Cambios estructurales en la familia}

La toma de decisiones con respecto a la SP podría tener un impacto a nivel de la estructura interna familiar, por lo que se exploraron cambios percibidos en el acompañamiento familiar, las relaciones familiares y la participación o no en la decisión (ver latabla 4).

Respecto al cuidado del paciente, nueve cuidadores refirieron que el acompañamiento durante el proceso de sedación no disminuyó y, por el contrario, aumentó debido a la percepción de mayor cercanía de la muerte. Solo un cuidador refirió poco acompañamiento por parte de la familia de su paciente debido a conflictos relacionales.

Con respecto al proceso de tomar la decisión, se encontró por un lado, que siete cuidadores percibieron que la familia participó en ésta, por sugerencia del médico. Por otro lado, tres cuidadores percibieron que el médico les informó sobre la sedación como la opción indicada para su familiar y ellos estuvieron de acuerdo.

Con relación a la percepción de los cuidadores sobre los cambios en las re- 


\section{Tabla 4. Cambios estructurales en la familia}

\begin{tabular}{ll}
\hline $\begin{array}{l}\text { Cuidado del } \\
\text { paciente }\end{array}$ & "Estuvo más acompañada y yo me quede más tiempo con ella, yo amanecí \\
la última noche con ella, porque nos dijeron que ya se estaba muriendo." & (CF8) \\
& "Pues en realidad, la familia de mi esposo estuvo muy ajena a la situación \\
& y yo sabía que podía o no esperar de ellos y que solo importábamos en ese \\
& momento mi esposo, mis hijos y yo." (CF5) \\
\hline Proceso de toma de & "El médico nos dijo en la mañana, hablen, discutan, tomen la decisión \\
decisión & que ustedes crean conveniente. Nos explicó muy bien como era el \\
& procedimiento. Inmediatamente tomamos la decisión." (CF8) \\
& "Fue el médico el que tomo la decisión, simplemente me informaron que \\
& la iban a sedar." (CF3) \\
\hline Cambios en las & "Ahora mi hermano y yo hemos estado muy unidos, siempre tuvimos vidas \\
relaciones familiares & muy diferentes, pero ahora que mi mamá no está yo estoy más cercano \\
& a él y le prometí a mi mamá que lo iba a cuidar y acompañar mucho \\
& más, entonces ahora nos hablamos todos los días y compartimos más. Y \\
& la familia de mi mamá esta también mucho más pendiente de nosotros \\
& ahora." (CF6) \\
& "Ha empeorado con la familia de él, ahora se han distanciado más de mis \\
& hijos y de mí también, eso es de parte y parte. La verdad es mejor así. Era \\
& más un vínculo por obligación, porque él estaba enfermo, él ya no está ya \\
se acabó todo." (CF5)
\end{tabular}

laciones familiares, nueve describieron "buenas relaciones familiares", refiriendo que estas no se afectaron durante el proceso de la sedación sino que se fortalecieron. Solo un cuidador describió relaciones distantes y conflictivas con la familia de su paciente, las cuales se deterioraron más luego del fallecimiento.

\section{Proceso de duelo}

Los pacientes candidatos a SP están en un proceso avanzado de su enfermedad, y su familia ha debido verse enfrentada a dicha realidad. Por lo tanto, se espera que tanto paciente como familia vivan un proceso de duelo que permita adaptarse a la situación y lograr un aprendizaje de su experiencia ante la enfermedad y la posible muerte. Por esta razón fue considerado importante indagar si la SP favorecía o no el proceso de duelo (ver respuestas en la tabla 5).
Se encontró que la sedación facilitó dicho proceso, ya que 9 de los cuidadores indicaron que la SP les ayudó en el proceso de duelo posterior a la muerte $y$ consideraron que si no se hubiese sedado a su familiar, la muerte podría haber sido dolorosa y traumática.

Con relación a la resolución de asuntos pendientes y la despedida como parte del proceso de duelo, nueve cuidadores reportaron no haber podido despedirse antes de iniciar la sedación, acto que realizaron durante la sedación por recomendación del personal asistencial. Solo un cuidador reportó haberse despedido antes del inicio de la sedación profunda y posteriormente.

Sobre el proceso de muerte como tal, nueve cuidadores la recuerdan como una buena muerte y una experiencia tranquila. Sin embargo, un cuidador recuerda la muerte como dolorosa y con sufrimiento. 


\title{
Tabla 5. Respuestas familiares ante el proceso de duelo
}

\author{
Facilitación del \\ "Si, está facilitando el proceso de todos. La ausencia es muy difícil pero el \\ proceso de duelo \\ comprender que ella fue quien murió y que nosotros seguimos y que ella tuvo \\ una buena muerte." (CF8)
}

Resolución

de asuntos

pendientes $y$

despedida

\begin{abstract}
"Si nos pudimos despedir de ella, hasta lo último le hablamos yo le decía mucho que se fuera tranquila que yo como hija mayor iba a estar muy pendiente de mi hermano y mis sobrinos y ella me movía la cabeza como diciéndome que estaba más tranquila. El poderle hablar me dio tranquilidad y a ella también, porque nosotras éramos muy apegadas y cuando yo le dije todo eso ella descanso y sintió que yo podía estar también tranquila. Igual para mí fue muy duro decírselo porque era soltarla pero si me dio más tranquilidad porque ella estaba apegada a la vida era por mí y eso le dio más tranquilidad." (CF8)

"Logre despedirme antes de la sedación profunda, lo abrace y hablamos muchas cosas que nunca antes habíamos hablado tan claramente, eso fue la última noche que él ya estaba muy mal y estaba desesperado porque le aumentarán la sedación y todos sabíamos que la dosis que se le iba a poner era ya para la sedación profunda." (CF7)
\end{abstract}

Proceso de muerte
"Tuvo una muerte hermosa, todos se fueron y yo me quede sola con él, las enfermeras lo cambiaron de posición, lo secaron porque había sudado mucho y lo pusieron muy lindo; uno viene haciendo muchas despedidas durante el proceso de enfermedad y el doctor fue muy insistente en que le habláramos mucho que nunca se sabía que palabras le podían ayudar a sentirse en paz y fue muy bonito porque yo lo hice, para mí esa conversación fue lo que le ayudo a morirse tranquilo." (CF10)

"No, un recuerdo imborrable, porque ver uno una agonía de esas, más o menos seis horas, un paciente tan desesperado. Porque ella murió muy asfixiada, muy desesperada. Eso es difícil de borrar, aquí lo recuerda uno con dolor, pero no con desespero. A nosotros nos ayuda que somos muy católicos (...) pero la recordamos muy tristes, pues murió muy desesperada." (CF1)

\section{DISCUSIÓN Y CONCLUSIONES}

Esta investigación tuvo como objetivo describir el impacto familiar de la SP en pacientes con enfermedad avanzada terminal ya fallecidos, según la percepción del cuidador principal. La discusión de los resultados encontrados se hará de acuerdo a las categorías preestablecidas.

Los resultados obtenidos en la categoría de conocimiento familiar de la SP coinciden con lo encontrado en la literatura, con respecto a la información dada por el personal de salud a las familias y las numerosas preocupaciones en torno al bienestar del paciente durante el proceso de SP y la necesidad continua de información y acompañamiento ante una experiencia desconocida ${ }^{(14)}$.

Resulta llamativo que en la literatura no se haya explorado qué tanto conocimiento o información previa tienen los familiares para enfrentarse a la SP, encontrando en esta investigación que la mayoría de los cuidadores no conocían esta alternativa. Según el Atlas Latinoamericano de Cuidados Paliativos, el desarrollo de los cuidados paliativos en nuestro país es medio-bajo, lo que posiblemente puede explicar este desconocimiento, pues es 
un servicio que se presta en niveles asistenciales especializados, dificultando la permeabilidad a la comunidad general ${ }^{(25)}$.

Es importante resaltar que en esta investigación se corroboran los hallazgos de la literatura en los que se indica que la mayor preocupación en las familias es no poder comunicarse con el paciente y tener insuficiente información y preparación para enfrentarse a los cambios físicos de su ser querido ${ }^{(10,15)}$. Sin embargo, la mayoría de los cuidadores del presente estudio evaluaron la experiencia de SP como positiva para el alivio del sufrimiento del paciente y la familia, lo que coincide con los reportado en la revisión sistemática de Bruinsma et al.(15).

Con relación a los cambios emocionales en la familia, y a diferencia de lo encontrado en otros estudios, la mayoría de los cuidadores refirieron sentir alivio ante la propuesta y este sentimiento continuó durante y después del proceso de sedación. De hecho, todos los cuidadores manifestaron que volverían a aceptar dicha opción terapéutica para otro familiar y para ellos mismos, si fuera el caso; incluyendo el cuidador que no percibió alivio por considerar que la sedación se aplicó tarde. Solo tres cuidadores reportaron malestar emocional y cuestionamientos relacionados con la toma de decisión. En ese sentido, en el estudio de Morita et al.(10) dicha preocupación fue una de las mayores generadoras de malestar emocional.

Las dinámicas y vivencias de las distintas etapas del ciclo vital de las familias se ven afectadas cuando uno de sus miembros experimenta una enfermedad terminal(26). Al momento de asumir y/o compartir la decisión y la responsabilidad de la SP se podrían producir cambios, alteraciones y desequilibrios mayores relacionados con el temor y desconocimiento de los beneficios del procedimiento. Sin embargo, no se han encontrado evidencias al respecto en la literatura. En el presente estudio se encontraron cambios estructurales en las tipologías familiares, pasando de familias nucleares a monoparentales por el fallecimiento de uno de los padres; por otro lado, las características de las familias de los cuidadores dan cuenta de sistemas que pudieron brindar soporte tanto al paciente como a los cuidadores. Con respecto al ciclo vital, el hecho de que la mayoría de familias estaban en etapa de nido acogedor (donde no se da la salida de los hijos) podría favorecer un soporte más numeroso y estable, facilitando la adaptación a la enfermedad y muerte ${ }^{(21)}$.

Según Fernández-Ortega ${ }^{(27)}$, el ciclo vital puede verse afectado por la consecución de las tareas propias de cada etapa y la posición de cada uno de sus miembros en sus roles. Los cuidadores entrevistados que refirieron mayor preocupación se encontraban en etapa de adolescencia; tal como lo plantea Navarro ${ }^{(21,28)}$, la enfermedad saca a la familia de su momento natural exigiéndole compatibilizar el cumplimiento de la tarea evolutiva con el cuidado de la enfermedad; ello conlleva el peligro de negación de la enfermedad o la detención en un momento evolutivo.

Ante el cuidado de un miembro, la familia debe buscar estrategias para organizarse y suplir las necesidades básicas de su paciente. Al respecto se encontró que nueve cuidadores no reportaron cambios ni disminución en el cuidado durante la sedación y, por el contrario, aumentó el número de acompañantes durante la hospitalización. En el estudio de Morita et al. ${ }^{(10)}$ algunas familias manifestaron no encontrar sentido en acompañar en este estado, sin embargo no hay reporte de abandono al paciente durante la sedación. Este resultado en nuestro estudio se conecta con el fortalecimiento de las relaciones familiares, percibidas por nueve cuidadores, quienes reportan luego del fallecimiento de su paciente, mayor cercanía y comunicación en la familia. 
La literatura reporta que quienes toman la decisión de SP son los pacientes $\mathrm{o}$, en su defecto, la familia ${ }^{(5,8,12)}$. Esta investigación encontró que siete cuidadores percibieron haber participado de ésta decisión y tres cuidadores percibieron que el médico solo comunicó dicha decisión; No obstante, todos los cuidadores estuvieron de acuerdo con la sedación como medio para aliviar el sufrimiento.

Ante la muerte de una persona, su sistema familiar se ve en la necesidad de adaptarse en diversos niveles de funcionamiento como lo son: la reorganización de sus sistemas comunicacionales, nuevas reglas, nuevas jerarquías y la redistribución de roles $^{(29)}$. Es posible pues hablar de una muerte simbólica para el sistema familiar, lo que genera una nueva familia que se ajusta a los cambios de su nueva realidad; posiblemente pueda sentirse amenazada su propia existencia como grupo familiar, lo cual puede generar mayor cercanía entre sus miembros e intensificación de sus relaciones; defendiéndose así como siste$\mathrm{ma}^{(23)}$. Esta investigación tuvo su mayor interés en explorar la percepción que tenía el cuidador principal sobre el proceso de duelo familiar y si la sedación lo facilitaba o no, puesto que la literatura al respecto es escasa. Se encontró que todos los cuidadores consideraron que la sedación estaba facilitando su proceso de duelo y percibieron que si no se hubiera sedado a su familiar, los últimos días hubieran sido dolorosos y traumáticos para toda la familia.

La mayoría de los cuidadores y sus familias recuerdan la muerte de su ser querido como tranquila y buena, pudiendose despedir y resolver asuntos pendientes de la familia hacia el paciente; En este sentido, el constructivismo social concibe el duelo como un proceso emocional $y$, por lo tanto, se relaciona con la manera como las personas construyen los acontecimientos que ocurren a su alrededor, lo cual va a depender de creencias y valores propios de la cultura de la cual hace parte ${ }^{(23)}$. Por lo tanto, la muerte que se avisa o que se espera, como ocurre con un familiar en SP, permite un espacio de preparación para cerrar historias y resolver conflictos y asuntos pendientes dentro de la familia, dando un nuevo valor a los elementos con los que la familia debe construir su realidad sin su ser querido ${ }^{(24)}$.

A modo de conclusión, las familias tienen necesidades particulares cuando alguno de sus miembros se encuentra bajo SP: información sobre los cambios físicos, sobre el bienestar de su paciente, qué siente o escucha cuando está bajo SP. Son numerosas las inquietudes de los familiares durante el proceso y es responsabilidad del equipo asistencial acompañar en este sentido. No obstante, a pesar de las dudas, temores y angustia ante el sufrimiento de su familiar, los cuidadores evaluaron la experiencia como positiva y satisfactoria, al disminuir el malestar emocional del paciente y de la familia.

\section{Limitaciones del estudio}

Este estudio empleó un muestreo por conveniencia, donde se excluyeron los familiares de pacientes que presentaran importante malestar emocional, con el fin de evitar situaciones iatrogénicas derivadas de la investigación. Sin embargo ello puede acarrear un sesgo de selección ya que no es posible saber qué tipo de impacto puede haber generado la SP en familias cuyos miembros hayan tenido dicho tipo de impacto. Asimismo, la mayoría de los participantes fue de sexo femenino, lo cual es coherente con la tendencia mundial que existe en el género de los cuidadores. No obstante, futuros estudios deberían incluir más población masculina para examinar sus percepciones acerca de la SP. 
Implicaciones para la práctica clínica

Los resultados del estudio permiten sugerir las siguientes recomendaciones al equipo asistencial para el acompañamiento durante la SP:

1. Presentar la opción de SP tempranamente durante proceso de enfermedad como alternativa para aliviar el sufrimiento y no solo cuando el paciente es candidato, para disminuir la angustia anticipada de pacientes y familiares.

2. Plantear la posibilidad de SP de manera empática y clara, facilitando la expresión de inquietudes y temores al respecto.

3. Establecer la diferencia entre SP y eutanasia, enfatizando en el objetivo de aliviar el sufrimiento y no causar la muerte.

4. Favorecer la toma de decisión conjunta entre el equipo asistencial y la familia, sin generar cargas de responsabilidad y estableciendo como meta el bienestar del paciente.

5. Hablar explícitamente de los posibles cambios físicos que el paciente puede presentar durante la SP, del tiempo aproximado de duración de la sedación y de los síntomas propios de la sedación, diferenciandolos del proceso de agonía.

6. Facilitar procesos de despedida y comunicación entre el paciente y la familia antes de iniciar la sedación; De no ser posible, reforzar en la familia el constante acompañamiento y brindarle espacios de desahogo emocional.

7. Brindar un acompañamiento que facilite un proceso de cuidado digno y favorezca el proceso de duelo anticipado, la resolución de necesidades espirituales y de malestar emocional antes, durante y después de la sedación.

\section{REFERENCIAS BIBLIOGRÁFICAS}

1. Pessini L. A filosofia dos cuidados paliativos: uma resposta da obstinação terapêutica. Em: Pessini L, Bertachini L, editores. Humanização e cuidados paliativos. 3. ed. São Paulo: Loyola, 2006.p.181-208.

2. Pessini L, Bertachini L. Nuevas perspectivas en cuidados paliativos. Acta Bioeth 2006;12:231-42. Doi: 10.4067/S1726569X2006000200012

3. Pessini L, Bertachini L. Humanização e cuidados paliativos. São Paulo: EDUNISC : Edições Loyola, 2004.

4. OMS. Cuidados paliativos [Internet]. Geneva: WHO; 2015 [Acceso 14 de junio de 2014]. Disponible en: http://www. who.int/cancer/palliative/es/

5. Sanz J. La sedación paliativa como lex artis en el cuidado del final de la vida. Psicooncologia 2008;5:265-77.

6. Anquinet L, Rietjens J, van der Heide A, Bruinsma S, Janssens R, Deliens L, et al. Physicians' experiences and perspectives regarding the use of continuous sedation until death for cancer patients in the context of psychological and existential suffering at the end of life. Psychooncology 2014;23:539-46. Doi: 10.1002/pon.3450

7. Anquinet L, Raus K, Sterck S, Smets T, Deliens L, Rietjens JA. Similarities and differences between continuous sedation until death and euthanasia - professional caregivers' attitudes and experiences: A focus group study. Palliat Med 2013;27:553-61. Doi: 10.1177/0269216312462272.

8. Engström J, Bruno E, Holm B, Hellzén O. Palliative sedation at end of life: a systematic literature review. Eur J Oncol Nurs 2007;11:26-35. Doi:10.1016/j. ejon.2006.02.007

9. Ten Have H, Welie JV. Palliative sedation versus euthanasia: an ethical assessment. J Pain Symptom Manage 2014;47:123-36. Doi: 10.1016/j.jpainsymman.2013.03.008 
10. Morita T, Ikenaga M, Adachi I, Narabayashi I, Kizawa Y, Honke Y, et al. Family experience with palliative sedation therapy for terminally ill cancer patients. J Pain Symptom Manage 2004;28:557-65.

11. Schröder PM. Impacto emocional y tratamiento psicologico durante la fase terminal. En: Die Trill M, editor. Psicooncología. Madrid: ADES Ediciones, 2003.p.631-45.

12. Organización Médica Colegial (OMC), Sociedad Española de Cuidados Paliativos (SECPAL). Guia de sedación paliativa. Madrid: 2011. Disponible en: https://www. cgcom.es/sites/default/files/guia_sedaccion_paliativa.pdf

13. Tschann JM, Kaufman SR, Micco GP. Family involvement in end-of-life hospital care. J Am Geriatr Soc 2003;51:835-40. Doi:10.1046/j.1365-2389.2003.51266.x

14. Van Dooren S, van Veluw HT, van Zuylen L, Rietjens JA, Passchier J, van der Rijt CC. Exploration of concerns of relatives during continuous palliative sedation of their family members with cancer. J Pain Symptom Manage 2009;38:452-9. Doi: 10.1016/j.jpainsymman.2008.11.011

15. Bruinsma SM, Rietjens JA, Seymour JE, Anquinet $L$, van der Heide $A$. The experiences of relatives with the practice of palliative sedation: A systematic review. J Pain Symptom Manage 2012;44:431-45. Doi: 10.1016/j.jpainsymman.2011.09.006

16. Waldrop DP, Kramer BJ, Skretny JA, Milch RA, Finn W. Final transitions: Family caregiving at the end of life. J PaIliat Med 2005;8:623-38. Doi:10.1089/ jpm.2005.8.623.

17. Koerner SS, Kenyon DB, Shirai Y. Caregiving for elder relatives: Which caregivers experience personal benefits/gains? Arch Gerontol Geriatr 2009;48:238-45. Doi: 10.1016/j.archger.2008.01.015

18. García JR. Los tiempos del cuidado: el impacto de la dependencia de los mayores en la vida cotidiana de sus cuidadores. Madrid: Imserso; 2010.

19. Krikorian-D A, Velez-A MC, González-T O, Palacio-G C, Vargas-G JJ. La experiencia de sufrimiento en cuidadores principales de pacientes con dolor oncológico y no oncológico. Av Enferm 2010;28:13-20.

20. Aoun SM, Kristjanson LJ, Currow DC, Hudson PL. Caregiving for the terminally ill: at what cost? Palliat Med 2005;19:5515. Doi:10.1191/0269216305pm1053oa

21. Navarro GJ. Enfermedad y familia: manual de intervención psicosocial. Barcelona: Paidós, 2004.

22. Fernández L, Rodríguez V. Intervenciones sobre problemas relacionados con el duelo para profesionales de atención primaria (I): el proceso de duelo. MEDIFAM 2002;12:218-25.

23. Parada-M LM. Duelo por muerte súbita desde el enfoque apreciativo: una opción de vida desde la pérdida. Diversitas 2007;3:55-66.

24. Worden JW. Grief counseling and grief therapy. 3. ed. New York: Springer, 1991.

25. Pastrana T, De Lima L, Pons JJ, Centeno C. Atlas de cuidados paliativos en Latinoamérica. Houston, TX: AHPC; 2013.

26. Harding R, List S, Epiphaniou E, Jones $\mathrm{H}$. How can informal caregivers in cancer and palliative care be supported? An updated systematic literature review of interventions and their effectiveness. Palliat Med 2012;26:7-22. Doi: 10.1177/0269216311409613

27. Fernández Ortega MÁ. El impacto de la enfermedad en la familia. Rev Fac Med 2009;47:251-4.

28. Navarro GJ. Terapia familiar con enfermos físicos crónicos. En: Navarro Góngora J, Beyebach M. Avances en terapia familiar sistémica. Buenos Aires: Paidós; 1995.p. 299-336.

29. Baider L. Cáncer y familia: aspectos teóricos y terapéuticos. Int J Clin Health Psychol 2003:505-20. 


\section{Anexo 1. Guía de entrevista}

1. ¿Cuál fue el diagnóstico que llevó a su familiar a una enfermedad avanzada?

2. ¿Qué tipo de tratamiento le realizaron?

3. ¿Le propusieron o le hablaron en algún momento de SP?

4. ¿Qué entendió por SP y de su objetivo?

5. ¿El personal sanitario le explicó de que se trataba la sedación y cómo lo hizo?, ¿Qué tan importante fue este acompañamiento?

6. ¿Qué sabía usted o su familia de la SP, antes de ser propuesta por el médico tratante?

7. ¿Qué expectativas tenía usted con relación a la sedación?, ¿Se cumplieron estas expectativas?

8. ¿Qué cambios observó usted en su paciente luego de ser aplicada la SP?, ¿Esperaba usted estos cambios o en algún momento le informaron que iban a ocurrir?

9. ¿Considera usted que la SP alivió el sufrimiento de su familiar?

10. ¿Teniendo como base ésta experiencia, si usted se viera enfrentado a una enfermedad avanzada, usted consideraría la opción terapéutica de la SP?

11. ¿Recomendaría usted a otra familia la opción terapéutica de la SP?

12. ¿Qué significó para usted la experiencia de la SP?

13. ¿Qué sintió usted cuando le hablaron de SP?

14. ¿Cómo percibió emocionalmente a su familia ante la decisión de la sedación?

15. ¿Alguno reaccionó diferente al resto de la familia?

16. ¿Cómo se sintieron usted y su familia durante el proceso de la sedación?

17. ¿Cómo se siente al día de hoy con la decisión de sedar a su familiar?

18. ¿Cómo distribuyeron las tareas de cuidado al paciente durante el proceso de hospitalización?

19. ¿Cómo fue el proceso de la toma de decisión para la SP?, ¿Quiénes participaron de esta decisión?

20. ¿La distribución de las tareas del cuidado al paciente en la hospitalización cambió durante la SP?, ¿Cómo cambió?

21. ¿Percibe usted a su familia distante o unida en sus relaciones?

22. ¿Esta forma de relacionarse, en que favoreció o dificultó la experiencia de sedación?

23. ¿Cómo describe ahora las relaciones familiares, después del fallecimiento?

24. ¿Cuáles cambios observó al interior de su familia durante el proceso de la SP?

25. ¿Teniendo en cuenta que usted y su familia en éste momento están realizando un proceso de duelo, considera que la opción de la sedación les está haciendo más fácil o difícil éste proceso?

26. ¿Cree usted que la SP les facilitó o dificultó el proceso de despedirse de su familiar?, ¿Se resolvieron asuntos pendientes?

27. ¿Qué miembros de la familia están presentando más dificultades en el proceso de duelo?, ¿Por qué?

28. ¿Cómo cree que su familia recuerda la muerte de su ser querido? 
